\title{
Impinging jets - a short review on strategies for heat transfer enhancement
}

\author{
Ilinca Nastase $^{1, *}$ and Florin Bode ${ }^{1,2}$ \\ ${ }^{1}$ CAMBI, Technical University of Civil Engineering in Bucharest, Building Services Faculty, 020396, Bucharest, Romania \\ ${ }^{2}$ Technical University of Cluj Napoca, Mechanical Engineering Faculty, Cluj-Napoca, Romania
}

\begin{abstract}
In industrial applications, heat and mass transfer can be considerably increased using impinging jets. A large number of flow phenomena will be generated by the impinging flow, such as: large scale structures, large curvature involving strong shear and normal stresses, stagnation in the wall boundary layers, heat transfer with the impinged wall, small scale turbulent mixing. All these phenomena are highly unsteady and even if nowadays a substantial number of studies in the literature are dedicated, the impinging jets are still not fully understood due to the highly unsteady nature and more over due to great difficulty of performing detailed numerical and experimental investigations.
\end{abstract}

\section{Introduction}

In industrial applications, heat and mass transfer can be considerably increased using impinging jets. Impinging jets can produce very high heat/mass transfer rates since they exhibit amongst the highest known levels of transfer capabilities for single phase flows, especially at low nozzle-to-plate distances [1, 2]. An oriented fluid flow versus a surface can transfer substantial amounts of mass and heat between that surface and the fluid. For example, compared with conventional convection cooling by confined flow parallel to the cooled surface, the use of jet impingement produces in increase in the heat transfer coefficients that are up to three times higher at a given maximum flow speed. Applications to engineering fields include turbine blade cooling [3], paper and fabric drying [4], furnace heating [5], tempering of glass and metal sheets $[6,7]$, food processing [8] and many others. Heat transfer enhanced by forced convection with impinging jets is known to yield high local and average heat transfer coefficients in the cooling of electronic components [9]. In this field, technological advance relies very much on the ability to dissipate extremely large heat fluxes [10]. Thus, interest in the present topic, from the standpoint of both theoretical and technical assessments, continues to be unquestioned.

The investigation tools, either experimental or numerical, have undergone tremendous developments which allow for a deeper comprehension of the involved phenomena. Since Martin [11] published in 1977 his pioneering review article on heat and mass transfer of impinging jets, several additional literature reviews have appeared [12-15]. In the case of the impinging jet, turbulence is generated by the flow itself and by possible external disturbances and varies significantly with the nozzle shape, the upstream conditions and the position within the jet [16]. The details of the nozzles, and of the upstream flow facility, is important in the design of heat transfer equipment in which turbulent jets impinge at relatively short nozzle-to-plate spacing $\mathrm{H} / \mathrm{D}$, but are secondary if impingement occurs beyond H/D $>8$ (Fig. $1)$.

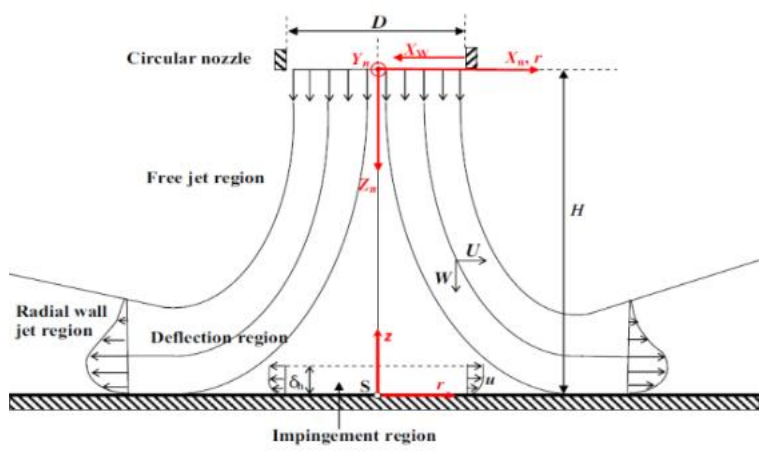

Fig. 1 Schematic description of impinging jet on a flat plate [17]

\section{Some aspects related to the physics of the impinging jets}

The commonly used designs of jet nozzle orifices are either circular nozzle, producing an axisymmetric velocity profile, or slitdiffusersproducing a wide thin jet mainly having a plane two-dimensional velocity profile.

The axisymmetric and plane free jets have been extensively studied in the past and their mean flow is described in reference works on free jets $[18,19]$. Other review works are available on their impinging counterparts [20-22]. 
A schematic representation of an axisymmetric impinging jet is represented in Fig.1. A fluid flow with uniform velocity issued from a circular nozzle and impinging orthogonally on a wallis considered in this case. As shown in Fig. 1, the flow of a submerged impinging jet elapses through several different regions, some of which may not exist according to the distance between the nozzle originating the jet and the impinging plate $\mathrm{H}$.

A jet emerges from a nozzle with characteristicmean and turbulent velocity and temperature profiles dependent upon the upstream flow conditions and nozzle shape[19]. For a pipe-shaped nozzle, the flow generally develops into the velocity profile typical of pipe flows with a certain turbulence, initialized upstream. For a flow generated by a differential pressure across a thin plate with an orifice, just downstream of this orifice a vena contractaphenomenon is produced, which is characterized by an initial flow having a flat velocity profile with low turbulence intensity. After exiting the nozzle, an impinging jet passes through a region where it is sufficiently far from the impingement surface and so behaves as a free submerged jet. Generally, the observation of the circular free jet emerging from a diffuser in an immobile environment, or itself animated with a velocity lower than the one the jet, shows a widening of the flow starting from the exit plane. It is common to distinguish three regions away from the jet outlet and on its axis: a potential core region, a settlement region, and a fully established region [19, 23$25]$. In the region of the potential core at the confluence of the two fluids, an annular mixing zone of increasing thickness is generated, delimiting a so-called potential cone within which the flow has the same conditions as those of the jet at its outlet (velocity, temperature, density ...). A geometric definition of the potential cone, also called potential core, would be given by the place where the longitudinal mean velocity $U$ would be $0.99 \mathrm{U} 0$, where U0 is the axial longitudinal velocity at the exit plane. Its length varies for a circular jet between 4 and 6 diameters of the nozzle [19, 23, 26].

The mixing layer around the annular potential cone is the seat of a strong shear. On either side of the potential core, the velocity decreases to join to the lower speeds of the environment. This connection is made by braking the velocity of the potential cone through the entrainement of the ambient fluid that mixes with the injected fluid[19]. In this zone, the average longitudinal components of velocity and of its turbulent component obey to similitude laws $[18,19,27]$. In addition, the turbulent energy is concentrated around the axis of the mixing layer where the radial gradients of the velocity are maximal. We find there vortices centered on the axis of the mixing layer whose size increases with the longitudinal distance $\mathrm{X}$. These vortices initially have a spatio-temporal coherence [28].

In the proximity of the plate, the axial velocity of the flow decays quickly and the jet is forced to undergo a sudden change of direction in a region called stagnation region.In the neighbourhood of the impinging point $S$ the flow spreads in radial directions parallel to the wall. A stagnation point with zero velocity exists on the wall in the intersection with the jet axis. The axial velocity component decreases and is transformed into an accelerated horizontal component. As a consequence, a high static pressure is created, over and above the wall, which transmits upstream the presence of the latter. Downstream the stagnation region, the flow spreads radially outward almost parallel to the impinged plate and develops into a semi-confined flow. Due to the jet finite width and the momentumexchange with quiescent surroundings and plate, the stagnation flow finally evolves into a wall jet flow (wall jet region).

As the plate gets closer to the nozzle exit, some of the abovereported regions are no longer present. First, the jet fully developedregion starts to disappear and then, the whole decaying jet regionmay not exist. If the plate lies within a distance of about two nozzlediameters, the high static pressure in the stagnation region hasa significant influence on the flow development up to the nozzle exit; therefore, also the usual quasi-conical shape of the core at small $\mathrm{H} / \mathrm{D}$ values, thevelocity profile of the jet does not have sufficient place to developand the arrival velocity is essentially uniform.

\section{Passive and active strategies for heat transfer enhancement}

Instability of the free shear layer downstream of the nozzle exit and turbulence development in the jet near field, are of fundamental importance in mass and heat transfer of impinging jet. Gardon and Akrifat [169], have studied the effect of turbulence on the heat transfer between two-dimensional jet and flat plate. It was shown that some seemingly anomalous heat-transfer phenomena can be explained as effects of the turbulence occurring in jets. Turbulence is generated by the jets themselves and by possible external disturbances and varies significantly with the nozzle shape, the upstream conditions and the position within the jet. The results presented by these authors suggest that details of the nozzles, and of the upstream flow facility, may be important in the design of heat transfer equipment in which turbulent jets impinge at relatively short nozzleto-plate spacing $\mathrm{H} / \mathrm{d}$, but are secondary if impingement occurs beyond $\mathrm{H} / \mathrm{d}=8$.

The great implications that vortical features can have on surface heat transfer rate and distribution motivated the flourishing of countless investigations devoted to passive and active strategies to enhance the heat flux between a jet and the impinged surface. Passive strategies are mainly based on the shape of the nozzle [29-31].

The geometries of the nozzles became progressively complex in the past decades in order to enhance the selfinduction of the asymmetric coherent structures [14-18]. The earliest studies conducted on rectangular jets revealed significant information on the structure and the dynamics of this type of jet flow. An interesting phenomenon was particularly discovered, namely, the switching of the major and minor axis of the jet. Krothapalli et al. [22] proposed a linear dependence between the distance from the nozzle to the first cross- 
over location and the nozzle aspect ratio. Later, Quinn [26] showed the existence of four pairs of counter rotating streamwise vortices next to the exit of the rectangular jet. These structures play an important part in the momentum transport. This author also reported a distortion of the jet's transverse geometry from a rectangular shape in the initial region to an elliptic shape in the far field. This phenomenon would be explained by the distortion of the azimuthal roll-up structures in addition to the nonuniform self-induction in the shear layer.

Furthermore, Hussain and Husain [15] showed that elliptic jets, unlike circular or plane jets, are characterized by a variation of the vortical structures azimuthal curvature, leading to non-uniform selfinduction and consequently to a complex threedimensional distortion that results in axis switching. They also emphasized a shorter potential core compared to the reference circular jet and higher levels of turbulent kinetic energy in this region. In a continuous effort to increase the spreading and mixing performance of the jet flows, other various methods have been explored. Rectangular and circular tabbed nozzles displayed a particularly improved mixing efficiency compared to the same geometries without mechanical tab intrusions [18, 28-30]. Each tab produces a pair of counter rotating streamwise vortices modifying the turbulent structure and increasing mixing in the jet flow.Geometrical manipulation of diffuser nozzles has been subsequently oriented to more complex shapes [31,32], and it has been suggested in recent work of $\mathrm{Hu}$ et al. [33-35], that the lobed nozzle is an extraordinary mixing device.

The previous observations have been exploited for imping jet applications. For instance, nozzles with chamfered outlets produce $20-30 \%$ increase of the heat transfer rate when compared to non-chamfered ones [32]. Variations from circular to elliptic nozzle exit section shapes are studied in [33], where, for an elliptic cross sectional area nozzle with aspect ratio equal to 4 , a maximum stagnation heat transfer $15 \%$ larger than for the circular configuration is found and a higher enhancement is found elsewhere. Other passive strategies are related to the use of vortex and turbulence generators in the nozzle geometry. In particular, Gao et al. [34] show that, for a nozzle-to-plate distance of 4 diameters, triangular tabs placed around a circular orifice (chevron nozzle) lead to an enhancement higher than $25 \%$ with respect to the round configuration for the same nozzle-to-plate distance. With respect to the circular impinging jet, a cross-shaped lobed jet [16, 35] attain the highest average heat transfer rate in the considered range of the Reynolds number compared with other devices in the literature, with very high heat transfer peaks (up to $75 \%$ enhancement at $\mathrm{H} / \mathrm{D}=2$ ).

Mixing and turbulence enhancement can be also promoted directly on the impinged surface with structure modification, for instance with fins having a triangular, square or rectangular cross section - an increase of the average Nusselt number value by $12-23 \%$ [36] can be produced. Surface roughness, in the form of cubes, produce heat transfer augmentation in the range of 8 $28 \%$ [37] while a dimpled surface is reported to reduce the heat transfer coefficient as compared to a smooth on [38]. Slot jet impinging on square and triangular ribbed walls are analysed in $[39,40]$. The reported increases may be due to combined effect of two factors, namely: the enhanced turbulence mixing caused by the rib distortion of the flow fields and the extension of the heat transfer surfaces (fin effect) provided by the ribs. In [41], vortex generators in the form of equilateral triangles are analysed and it is observed that the heat transfer coefficient between the impinging jet and the target plate is sensitive to the shape of the fin with an increase of the heat transfer coefficient up to $77 \%$. The augmentation of the heat transfer for the surfaces vortex generators is higher than that of the finned surfaces.

The active control methods generally use the acoustic [42-46] or mechanical [47-52] excitation to the exit plane of the flow, which favorsunder certain conditions, the amplification of the instabilities and the appearance of large highly energetic vortex structures that are generating mixing enhancement.Liu and Sullivan [53] studied the forcing of the impinging jet with flow visualization as well as velocity and heat transfer spectra from hot wire and hot-film sensors. Both heat transfer enhancement and reduction are found and are related to the development of largescale vortical structures in the wall jet region. When the excitation frequency is close to the natural frequency of the impinging jet, the initiated intermittent vortex pairing produces a chaotic lump eddy which contains a great deal of the small scale random turbulence which enhances the local heat transfer. Once the forcing is nearthe sub-harmonic of the natural frequency, stable vortex pairing is promoted. The strong large scale well organized vortices, formed after the stable pairing, induce the unsteady separation of the wall boundary layer and, hence, lead to the local heat transfer reduction.

\section{Advanced experimental methods and perspectives}

A large number of flow phenomena will be generated by the impinging flow, such as: large scale structures, large curvature involving strong shear and normal stresses, stagnation in the wall boundary layers, heat transfer with the impinged wall, small scale turbulent mixing. All these phenomena are highly unsteady and even if nowadays a substantial number of studies in the literature tried to illuminate this matter, the impinging jets are still not fully understood due to the highly unsteady nature and more over due to great difficulty of performing detailed numerical and experimental investigations [62].

There are several studies on the mass transfer between a plate and an impinging jet using limiting diffusion current technique. The study of Chin and Tsang[54] considered the mass transfer between an impinging jet and a circular disk electrode located in the stagnation region of the flow. They found that the electrode possesses a property of "uniform accessibility" to the diffusing species if the electrode radius is less than one nozzle diameter for the turbulent jet and half a 
nozzle diameter for the laminar jet. The authors have presented semi empirical correlations in both laminar and turbulent flow regimes for nozzle-to-plate distances from 0.2 to 6 nozzle diameters. The wall jet region was found to begin approximately at a radial distance of four nozzle diameters.

For the fluid flow measurement, a suitable technique is Particle Image Velocimetry (PIV). Due to threedimensional nature of the envisaged configurations (special geometries of the nozzles, impacting surface modification) the recent developments in the volumetric optical techniques such as tomographic PIV provide a suitable approach for the fluid flow analysis. Volumetric PIV is based on the double-pulsed laser illumination of a fluid volume seeded with particles. The particles distribution is reconstructed from multiple camera views and then the volumetric flow field is obtained through volume cross-correlation which allow for the computation also of derivative quantities giving a detailed description of vortex and turbulence dynamics [34]. Due to the laser scattering at the wall surface, velocity measurements with PIV method very close to the impinging surface are neither usually possible nor reliable. In this case, the electrodiffusion method (ED) is the most reliable method for measuring the wall shear rate [35]. The ED method is based on the measurement of the limiting diffusion current on a working electrode (probe) [36]. This technique has been developed for measuring the average rates of mass transfer and their fluctuations on a wall. The transfer area consists of a small polarized electrode flush mounted in the wall. An electrochemical redox reaction whose rate is limited by mass transfer is employed. ED measurements of the wall shear were first applied by Vallis et al. [37].

Yapici et al. [55] measured the surface distributions of the local shear stress values for a fully developed turbulent velocity profile, round, submerged jet, impinging on a flat surface. The results showed that the normalized local shear stresses values decreased with increasing Reynolds number and had a tendency to converge at higher Reynolds numbers. Alekseenko and Markovich [56] measured wall shear stresses of circular jet at a Reynolds number of 41600 and for nozzle-toplate distances from 2 to 8 nozzle diameters while Baleras et al. [57] studied the effect of the normal velocity on the segment currents of a three-segment circular probe in the vicinity of stagnation point at Reynolds number 640 . No study on the wall shear stress and mass transfer for a lobed impinging jet using the electrodiffusion technique was proposed until our paper[16]. Since the lobed orifice jet is characterized by intensified vortical dynamics and turbulent kinetic energy in its initial region, it is believed that such a complex jet flow will also display enhanced mass transfer when considered in impinging configuration. In the following are presented results from our article [16]. In this study we compare, in terms of mass transfer rates, a cross-shaped orifice nozzle to a reference convergent circular nozzle having close equivalent diameter. The knowledge issued from previous analysis of the near field of a cross-shaped orifice jet in free air-air jet configuration $[40,46]$, is used in an attempt to gain insight into the differences observed for the two studied impinging jets. Indeed, different variations of the radial distribution of wall shear rates and of the mass transfer at the stagnant point were obtained with the lobed nozzle flow relatively to the flow generated by the classical circular convergent nozzle. The wall shear rate of the impinging jets on a flat plate was measured by electrodiffusion technique and mass transfer was calculated from these values in the impingement region.

The difficulty of the experimental investigation arise from fact that flow field is extremely sensitive to external perturbations and hence the impossibility of introducing probes for measuring various quantities of the flow, the flow field being strongly influenced by these probes. In this case it is recommended the usage of nonintrusive research methods. The effect of the impinging jet in the heat and mass transfer for the studied flow is strongly dependent of the wall shear rate [77], hence by measuring this quantity, we can measure the imp act of the impinging jet. Also, the wall shear rate is influenced by the behavior of the flow. Therefore, in order to have a clear image of the impinging jet, we must determine in the same time the flow field and the wall shear rate.

In a first attempt of combining flow velocity and wall shear rate measurements El Hassan et al. [41, 42] performed simultaneously time resolved PIV and ED measurements. They have shown that the instantaneous wall shear stress is strongly dependent on the vortex dynamics. The interaction between primary and secondary structures, and their pairing process with a tertiary preceding structure, affects the ejection process near the wall and subsequently the wall shear stress variation. Recently, the team coordinated by Meslem [43] performed non-synchronized Time Resolved Tomo PIV and ED measurements comparing three circular jets: a convergent nozzle jet, a hemispherical nozzle jet and an orifice plate jet. A link between the wall shear stress and the mass transfer was revealed by the authors. The wall shear rate and the mass transfer were found to be in a close relationship with the near field flow features, themselves affected by the nozzle geometry. The instantaneous PIV fields indicated the formation of secondary vortices in the region where a secondary peak in local mass transfer emerges. The level of this secondary peak was found to be sensitive to the nozzle shape. The limited spatial resolution imposed by the used TR cameras in Tomo PIV configuration did not allowed the investigation of other geometries of nozzles. The vector fields were obtained by performing multipass direct cross-correlation using a final interrogation volume of $64 \times 64 \times 64$ voxels $(0.28 \mathrm{D} \times 0.28 \mathrm{D} \times 0.28 \mathrm{D})$. The intricate threedimensionalvortical characteristics of lobed jets for instance would not be capable of being captured by this configuration.

\section{Conclusions and perspectives}

In industrial applications, heat and mass transfer can be considerably increased using impinging jets.A large number of flow phenomena will be generated by the 
impinging flow, such as: large scale structures, large curvature involving strong shear and normal stresses, stagnation in the wall boundary layers, heat transfer with the impinged wall, small scale turbulent mixing. All these phenomena are highly unsteady and even if nowadays a substantial number of studies in the literature are dedicated, the impinging jets are still not fully understood due to the highly unsteady nature and more over due to great difficulty of performing detailed numerical and experimental investigations. If we want to gain knowledge about these fundamental phenomena, to understand how to control them for each practical application, we have to find methods that are adapted to our goal. We believe that the first step in this way is to perform classical volumetric PIV measurements allowing a much higher spatial resolution to capture the three-dimensional characteristics of more complicated impinging jet configurations. These measurements have to be synchronized with ED measurements. In addition, 3D LDV measurements triggered by the analogical ED signal will represent a useful, complementary, high resolution tool, allowing investigating specific regions of interests in the flows. Further understanding is needed about the vortex dynamics and breakdown as well as on the influence of streamwise vorticity. These issues necessarily call for the improvement of inspection capabilities of certain experimental techniques such as ED, IR thermography and volumetric PIV.

Advanced passive strategies such as the use of the lobed nozzles or structured surfaces are not sufficiently studied to provide higher cooling/heating efficiency. Most of the above mentioned issues have to be investigated not only from the experimental point of view but also from the numerical one with further refined methods. The nowadays available computing power allows to perform such theoretical studies without any major difficulties and with much less effort than the experimental ones. Nevertheless, it is obvious that experiments have an indispensable role since the development and validations of theories, models and algorithms still rely on the quality of experiments and call for even more challenging measurement.

\section{Acknowledgement}

This work was supported by the grant of the Romanian National Authority for Scientific Research, CNCS, UEFISCDI, PHANTOM - Passive flow control for heat and mass transfer enhancement of impinging jets PN-III-P4-ID-PCE-2016-0758

\section{References}

1. Narayanan, V., J. Seyed-Yagoobi, and R.H. Page, An experimental study of fluid mechanics and heat transfer in an impinging slot jet flow. International Journal of Heat and Mass Transfer, 2004. 47: p. 18271845 .

2. Baydar, E. and Y. Ozmen, An experimental and numerical investigation on a confined impinging air jet at high Reynolds numbers. Applied Thermal Engineering, 2005. 25: p. 409-421.
3. Han, B. and R.J. Goldstein, Jet-impingement heat transfer in gas turbine systems, in Annals of the New York Academy of Sciences. 2001. p. 147-161.

4. Polat, S., Heat and mass transfer in impingement drying. Drying Technology, 1993. 11(6): p. 1147-1176.

5. Viskanta, R., Heat transfer to impinging isothermal gas and flame jets. Experimental Thermal and Fluid Science, 1993. 6(2): p. 111-134.

6. Gardon, R. and J.C. Akfirat, Heat transfer characteristics of impinging two-dimensional air jets. J. Heat Transfer, 1966. 88: p. 101-108.

7. Gardon, R. and J. Cobonpue, 1962: p. 454-460.

8. Sarkar, A., et al., Fluid flow and heat transfer in air jet impingement in food processing. Journal of Food Science, 2004. 69(4): p. CRH113-CRH122.

9. Fabbri, M., S. Jiang, and V.K. Dhir, A comparative study of cooling of high power density electronics using sprays and microjets. Journal of Heat Transfer, 2005. 127(1): p. 38-48.

10. Anwarullah, M., V.V. Rao, and K.V. Sharma, Experimental investigation for enhancement of heat transfer from cooling of electronic components by circular air jet impingement. Heat and Mass Transfer, 2012. 48(9): p. 1627-1635.

11. Martin, H., Heat and Mass Transfer between Impinging Gas Jets and Solid Surfaces, in Advances in Heat Transfer. 1977. p. 1-60.

12. Carlomagno, G.M. and A. Ianiro, Thermo-fluiddynamics of submerged jets impinging at short nozzleto-plate distance: A review. Experimental Thermal and Fluid Science, 2014. 58( ): p. 15-35.

13. Hall, J.W. and D. Ewing, On the dynamics of the large-scale structures in round impinging jets. Journal of Fluid Mechanics, 2006. 555: p. 439-458.

14. Harmand, S., et al., Review of fluid flow and convective heat transfer within rotating disk cavities with impinging jet. International Journal of Thermal Sciences, 2013. 67: p. 1-30.

15. Polat, S., et al., Numerical flow and heat transfer under impinging jets: A review. Annual Review of Numerical Fluid Mechanics and Heat Transfer, 1989. 2: p. 157-197.

16. Kristiawan, M., et al., Wall shear rates and mass transfer in impinging jets: Comparison of circular convergent and cross-shaped orifice nozzles. International Journal of Heat and Mass Transfer, 2012. 55(1-3): p. 282-293.

17. Meslem, A., et al., Flow dynamics and mass transfer in impinging circular jet at low Reynolds number. Comparison of convergent and orifice nozzles. International Journal of Heat and Mass Transfer, 2013. 67: p. 25-45.

18. Abramovich, G.N., The theory of turbulent jets. 1963, Cambridge, Massachusets: MIT Press. 668.

19. Rajaratnam, N., Turbulent jets. 1976, Amsterdam, Netherlands: Elsevier Scientific Publishing Company.

20. Martin, H., Heat and mass transfer between impinging gas jets and solid surfaces. Advances Heat Transfer, 1977. 13: p. 1-60.

21.Jambunathan, K., et al., A review of heat transfer data for single circular jet impingement. International Journal of Heat and Fluid Flow, 1992. 13: p. 106-115. 
22. Carlomagno, G.M. and A. Ianiro, Thermo-fluiddynamics of submerged jets impinging at short nozzleto-plate distance: A review. Experimental Thermal and Fluid Science, 2014. 58(Supplement C): p. 15-35.

23. Awbi, H.B., Ventilation of Buildings. 1991, London, U.K.: E\&FN SPON. 313.

24. ASHRAE, ASHRAE Handbook Fundamentals. 1993, Atlanta: American Society of Heating, Refrigerating and Air Conditioning Engineering. Chap. 31 .

25.Tuve, G.L., Air velocities in ventilating jets, ASHVE Research Report No.1476, in ASHVE Transactions, 58. 1953. p. 261-283.

26. Oosthuisen, P.H. An experimental study of low Reynolds number turbulent circular jet flow. in ASME Applied Mechanics, Bioengineering and Fluids Engineering Conference,. 1983. Houston, U.S.A.

27. Rajaratnam, N. and B.S. Pani. Turbulent compound annular shear layers. in Proceedings ASCE, J. Hydraulics Division. 1972.

28. Davies, P.A.O.L., M. Fischer, and M.J. Barrat, The caracteristics of the turbulence in the mixing region of a round jet. Journal of Fluid Mechanics, 1963. 15: p. 337367.

29. Chaudhari, M., B. Puranik, and A. Agrawal, Effect of orifice shape in synthetic jet based impingement cooling. Experimental Thermal and Fluid Science, 2010. 34(2): p. 246-256.

30. Garimella, S.V. and B. Nenaydykh, Nozzlegeometry effects in liquid jet impingement heat transfer. International Journal of Heat and Mass Transfer, 1996. 39(14): p. 2915-2923.

31.Pan, Y., J. Stevens, and B.W. Webb, Effect of nozzle configuration on transport in the stagnation zone of axisymmetric, impinging free-surface liquid jets. Part 2. Local heat transfer. Journal of Heat Transfer, 1992. 114(4): p. 880-886.

32. Brignoni, L.A. and S.V. Garimella, Effects of nozzle-inlet chamfering on pressure drop and heat transfer in confined air jet impingement. International Journal of Heat and Mass Transfer, 2000. 43(7): p. 11331139.

33.Lee, J. and S.J. Lee, The effect of nozzle aspect ratio on stagnation region heat transfer characteristics of elliptic impinging jet. International Journal of Heat and Mass Transfer, 2000. 43(4): p. 555-575.

34.Gao, N., H. Sun, and D. Ewing, Heat transfer to impinging round jets with triangular tabs. International Journal of Heat and Mass Transfer, 2003. 46(14): p. 2557-2569.

35.Bode, F., A. Meslem, and C. Croitoru, Numerical simulation of a very low Reynolds cross-shaped jet. Mechanics, 2013. 19(5): p. 512-517.

36. Hansen, L.G. and B.W. Webb, Air jet impingement heat transfer from modified surfaces. International Journal of Heat and Mass Transfer, 1993. 36 p. 989-997.

37. Chakroun, W.M., A.A. Abdel-Rahman, and S.F. AlFahed, Heat transfer augmentation for air jet impinged on a rough surface. Applied Thermal Engineering, 1998. 18(12): p. 1225-1241.
38. Ekkad, S.V. and D. Kontrovitz, Jet impingement heat transfer on dimpled target surfaces. International Journal of Heat and Fluid Flow, 2002. 23(1): p. 22-28.

39. Gau, C. and C.C. Lee, Impingement cooling flow structure and heat transfer along rib-roughened walls. International Journal of Heat and Mass Transfer, 1992. 35(11): p. 3009-3020.

40.Gau, C. and I.C. Lee, Flow and impingement cooling heat transfer along triangular rib-roughened walls. International Journal of Heat and Mass Transfer, 2000. 43(24): p. 4405-4418.

41.Nakod, P.M., S.V. Prabhu, and R.P. Vedula, Heat transfer augmentation between impinging circular air jet and flat plate using finned surfaces and vortex generators. Experimental Thermal and Fluid Science, 2008. 32(5): p. 1168-1187.

42.Zaman, K.B.M.Q. and A.K.M.F.Hussain, Vortex pairing in a circular jet under controlled excitation. Part 1. General response. Journal of Fluid Mechanics, 1980. 101(3).

43.Hussain, F. and H.S. Husain, Elliptic jets. Part1. Characteristics of unexcited and excited jets. Journal of Fluid Mechanics, 1989. 208: p. 257-320.

44.Zaman, K.B.M.Q., Axis switching and spreading of an asymmetric jet: the role of coherent structure dynamics. Journal of Fluid Mechanics, 1996. 316(1): p. $1-27$.

45.Lai, J.C.S., Turbulence suppression in an elliptic jet. International Journal of Heat and Fluid Flow, 1992. 13(1).

46.Lin, Y.T., et al., Investigation on the mass entrainement of an acoustically controlled elliptic jet. International Communications in Heat and Mass Transfer, 1998. 25(3).

47. Wiltse, J.M. and A. Glezer, Manipulation of free shear flows using piezoelectric actuators. Journal of Fluid Mechanics, 1993. 249(261-285).

48.Parekh, D.E., et al., Innovative jet flow control : Mixing enhancement experiments. AIAA Paper 960808, 1996.

49. Suzuki, H., N. Kasagi, and Y. Suzuki, Active control of an axisymmetric jet with distributed electromagnetic flaps actuators. Experiments in Fluids, 2004. 36(498-509): p. 1-43.

50.Davis, M.R., Variable control of jet decay. AIAA Journal, 1982. 20(5).

51.Denis, S., Contrôle du developpement des couches de mélange axisymétriques subsoniques par jets impactant. 2000, Université de Poitiers. p. 280.

52. Collin, E., Etude de l'injection radiale de fluide dans une couche de mélange annulaire supersonique. 2001, Université de Poitiers. p. 282.

53.Liu, T. and J.P. Sullivan, Heat transfer and flow structures in an excited circular impinging jet. International Journal of Heat and Mass Transfer, 1996. 39(17): p. 3695-3706.

54.Chin, D.T. and C.H. Tsang, Mass transfer to an impinging jet electrode. Journal of the Electrochemical Society, 1978.

55. Yapici, S., et al., Surface shear stress for a submerged jet impingement using electrochemical 
technique. Journal of Applied Electrochemistry, 1999. 29: p. 185-190.

56. Alekseenko, S.V. and D.M. Markovich, Electrodiffusion diagnostics of wall shear stresses in impinging jet. Journal of Applied Electrochemistry, 1994. 24: p. 626-631.

57.Baleras, F., et al., A three-segement electrodiffusion probe in axisymmetric flow with stagnation and separation. Journal of Applied Electrochemistry, 1994. 24: p. 676-684. 